\title{
Review
}

Journal of Innate

Immunity
J Innate Immun 2017;9:441-455

DOI: $10.1159 / 000477419$
Received: April 18, 2017

Accepted after revision: May 8, 2017

Published online: July 12, 2017

\section{Update of Sepsis in the Intensive Care Unit}

\author{
Kelly Roveran Genga ${ }^{a} \quad$ James A. Russell ${ }^{a} b$ \\ ${ }^{a}$ Centre for Heart Lung Innovation and ${ }^{b}$ Department of Medicine, St. Paul's Hospital, University of \\ British Columbia, Vancouver, BC, Canada
}

\section{Keywords}

Sepsis - Septic shock · Early treatment · Albumin .

Transfusion · Renal therapy

\begin{abstract}
Sepsis, the most common cause of admission to an intensive care unit (ICU), has had an increased incidence and prevalence over the last years with a simultaneous decrease in its short-term mortality. Sepsis survivors are more frequently discharged from hospital and often experience long-term outcomes such as late mortality, immune dysfunction, secondary infections, impaired quality of life, and unplanned readmissions. Early recognition and management of sepsis have challenged emergency care and critical care physicians and nurses. New sepsis definitions were produced and the Surviving Sepsis Campaign (SSC) 2016 was updated recently. Although hospital readmissions after sepsis are common, associated risk factors and how to manage patients who survive an episode of sepsis still need clarification. The immune dysfunction caused by sepsis/septic shock is complex, persistent, affects inflammatory and anti-inflammatory systems, and might be associated with long-term outcomes of sepsis. Several randomized controlled trials (RCT) that analyzed new (and old) interventions in sepsis/septic shock are discussed in this review in parallel with the SSC 2016 recom-
\end{abstract}

\section{KARGER}

(ㅇ 2017 S. Karger AG, Basel

E-Mail karger@karger.com

www.karger.com/jin mendations and other guidelines when relevant. RCTs addressing incidence, treatment, and prevention of important sepsis-associated organ dysfunction such as the acute respiratory distress syndrome, acute kidney injury, and brain dysfunction are highlighted. Finally, we briefly discuss the need for novel targets, predictive biomarkers, and new designs of RCTs in sepsis.

(c) 2017 S. Karger AG, Basel

\section{Introduction}

The main purpose of this review is to consider, summarize, and discuss key studies in sepsis in 2016. We address studies published on the epidemiology of sepsis, readmission and quality of life after sepsis, immune dysfunction and secondary infections during and after sepsis, the new sepsis 3.0 definition, and then a series of key randomized controlled trials (RCTs). RCTs assessed potentially important interventions including: antimicrobial strategies, early goal-directed therapy (EGDT), vasopressors, albumin administration, and corticosteroids. We then discuss relevant RCTs in conditions that complicate sepsis such as the acute respiratory distress syndrome (ARDS).
Dr. James A. Russell

Centre for Heart Lung Innovation, St. Paul's Hospital

1081 Burrard Street

Vancouver, BC V6Z 1 Y6 (Canada)

E-Mail Jim.russell@ hli.ubc.ca 


\section{The Global Burden of Sepsis}

The incidence and prevalence of sepsis have increased globally over the last years [1-3]. Sepsis is the most frequent cause of admission to an intensive care unit (ICU), the most common cause of death in ICU [4], and a very common cause of hospital readmission in sepsis survivors [5-12], and has been recently reported as the final common pathway to death from infection [13]. The Global Burden of Disease [14] described infection as the cause of death of more than 10 million people per year, and sepsis affects between 3 and 10 per 1,000 people annually in high-income countries [15]. Despite that, given the promotion of an early recognition of sepsis and associated increases in health care reimbursements for a sepsis diagnosis, the true incidence of sepsis may be overestimated [16]. Additionally, the tools used for measuring sepsis incidence (analysis of insurance claims data and codes for sepsis, organ dysfunction, and/or infection) are not entirely reliable [16], and sepsis epidemiological studies are mostly from high-income countries, being scarce in low- and middle-income ones [15]. For these reasons, the interpretation of sepsis incidence and prevalence data by researchers, policy makers, and critical care physicians requires caution.

Despite the existence of updated sepsis/septic shock guidelines and bundles [17], which recommend early diagnosis and prompt institution of therapy aiming to prevent progression to organ(s) dysfunction(s), this syndrome continues to be a challenge worldwide. Progressive improvements in the management of sepsis have led to decreased mortality rates over the last decades (about 20$30 \%)[18,19]$ and increased complexity of care, and hence the costs associated with sepsis care remain high $[15,20]$.

\section{Increased Hospital Readmissions and Decreased Quality of Life after Sepsis}

The progressive increase in the number of sepsis and septic shock survivors emphasizes the long-term consequences of sepsis such as cognitive dysfunction and functional disabilities [21], psychiatric morbidity [22], decreased health-related quality of life [23], unplanned hospital readmissions $[10,24]$, and late mortality $[12,25]$.

\section{Hospital Readmissions}

The relationship between index sepsis hospitalization and unplanned hospital readmissions was demonstrated by Sun et al. [24] in a retrospective cohort study in the
USA. The study demonstrated that unplanned hospital readmissions after an episode of sepsis are common, and that infection was the commonest reason for readmission. Factors independently associated with hospital readmission were use of total parenteral nutrition, duration of antibiotics, prior hospitalizations, and lower hemoglobin at discharge. Interestingly, around half of all infection-related readmissions were readmitted at the emergency department with recurrent sepsis.

The 30-day readmission rates after sepsis were also evaluated by Mayr et al. [10] in a cohort that represents $49 \%$ of the US population. Among more than 1 million patients with index admissions due to medical reasons, and who had unplanned readmission within 30 days, sepsis was the most common and most expensive (evaluated by mean cost per readmission) reason for readmission, and was associated with the longest length of stay in the hospital when compared with acute myocardial infarction, pneumonia, heart failure, and chronic obstructive pulmonary disease.

These studies $[10,24]$ reinforce that more research is necessary to (1) understand better the risk factors associated with readmission after sepsis, (2) identify strategies to decrease the risk of readmission after sepsis, initially with observational cohort studies with propensity matching of readmitted and nonreadmitted patients, and then (3) prospective RCTs of interventions to ultimately define cost-effective interventions in sepsis survivors that decrease readmission rates after hospital discharge.

\section{Quality of Life}

Quality of life is frequently altered after sepsis $[21,22]$. However, it is unknown whether more intensive intervention improves quality of life after sepsis. Therefore, an RCT of usual sepsis aftercare versus a well-defined primary care-based intervention was conducted in Germany [26]. The two groups were compared with respect to the change in mental health-related quality of life evaluated between ICU discharge and 6 months thereafter; surprisingly, no differences between treatment groups was demonstrated in mental quality of life.

One challenge in studies of quality of life after sepsis is that the quality of life before sepsis is almost always not measured quantitatively using rigorous scoring systems but is only available by patient or family recall. So it is almost impossible to really convincingly show how much quality of life has changed after sepsis. Furthermore, studies analyzing quality of life after sepsis find that sepsis survivors have a wide range of sequelae after sepsis evaluated by heterogeneous methods that are often difficult to
442

J Innate Immun 2017;9:441-455

DOI: $10.1159 / 000477419$
Genga/Russell 
interpret. Additionally, it is unknown which instrument is the most appropriate for measurements of quality of life in septic patients. Finally, it remains uncertain whether a primary care intervention after sepsis will improve mental health-related quality of life $[26,27]$.

\section{Importance of Sepsis-Associated Immune Dysfunction: Long-Term Outcomes and Secondary Infections after Sepsis Admission}

It has been postulated - and shown in some studies [28] - that sepsis is associated with persistent dysfunction of immune and inflammatory systems. That may be one reason of why so many immune and inflammation modulation RCTs in sepsis have failed to improve survival [29].

\section{Immune Dysfunction}

The immune dysfunction of sepsis persists even at hospital discharge after clinical recovery, involves both innate immune dysregulation and adaptive immune suppression, and is complicated by simultaneous participation of the inflammatory and the anti-inflammatory responses. The innate and adaptive immune systems and the inflammatory and anti-inflammatory responses may fluctuate and conflict. These complex interactions likely play an important role in the recurrent, secondary, and nosocomial infections, and other long-term outcomes of sepsis, such as hospital readmissions and late mortality [29]. To date, we are unaware of RCTs that compare different immune and inflammatory-modulating interventions in sepsis survivors after discharge.

Secondary Infections after Sepsis Admission and Impaired Immune Function

The pathologic state of immune suppression present in sepsis is associated with the subsequent development of ICU-acquired nosocomial infections such as ventilator-associated pneumonia, urinary tract infection, catheter-associated bacteremia, antibiotic-associated diarrhea, and Clostridium difficile enterocolitis [30]. However, it is unclear how much nosocomial infections contribute to subsequent morbidity and mortality. A prospective observational study for the determination of the clinical and host genomic characteristics of ICU-acquired infections in critically ill patient conducted by van Vught et al. [31] demonstrated that the attributable mortality fraction of nosocomial infections contributed only a $2 \%$ absolute increase to overall mortality. In an exploratory analysis, leukocytes from patients who developed a secondary

Update of Sepsis in the ICU infection had a gene expression profile (at the time of ICU-acquired infection) that reflected impaired glycolysis and gluconeogenesis. Despite the limitations associated with such an exploratory analysis, this finding supports the hypothesis that secondary infections emerge due to acquired immune dysfunction, because leukocyte glycolysis is fundamental for energy supply and the inflammatory response of immune cells.

\section{New Sepsis Definitions (Sepsis-3)}

Importance of the Sequential Organ Failure

Assessment (SOFA) Score, Creation of Quick SOFA,

Inadequacy of Systemic Inflammatory Response

Syndrome Criteria, and Redundancy of the "Severe

Sepsis" Term

Singer et al. [32] updated the former sepsis definitions from 1992 [33] and 2003 [34] through an expert consensus process, based in part on the evidence from very large, multicenter derivation and validation cohorts in which the new definitions were derived and validated by Seymour et al. [35] and Shankar-Hari et al. [36], respectively. Sepsis-3 findings, strengths, and concerns associated with the new definitions of sepsis are described in Table 1. It is uncertain whether, when, and to what extent the new definitions will be used in new trials of therapies in sepsis.

\section{RCTs, Meta-Analyses, and Major Retrospective Studies in Sepsis}

\section{Antimicrobials}

Some evidence exists that continuous infusion (CI) of antimicrobials is better than the usual intravenous intermittent bolus (IB) administration, but large trials of efficacy have been lacking. Recently, CI and IB dosing of $\beta$-lactams were compared in patients with severe sepsis not receiving renal replacement therapy (RRT) in a twocenter RCT [37] conducted in Malaysia. Patients receiving CI had statistically significantly higher clinical cure rates (at 14 days after cessation of antibiotics), better pharmacokinetic/pharmacodynamic target attainment, and greater ventilator-free days than the IB group, whereas no differences were found between groups in ICU-free days, and 14- and 30-day survival. The novelty of this study was that it replicated similar findings from others $[38,39]$ in a distinct geographic region.

The Surviving Sepsis Campaign (SSC) 2016 guidelines [17] recommend that the doses of antibiotics should be

J Innate Immun 2017;9:441-455 DOI: $10.1159 / 000477419$ 
Table 1. New sepsis definitions: findings, strengths, and concerns

\section{Major findings from large, multicenter sepsis cohorts}

Seymour et al.

SOFA score $\geq 2$ represents an overall mortality risk of approximately $10 \%$ in patients with suspected infection in a general hospital

The sepsis screening tool "qSOFA" was defined = THAM - tachypnea (respiratory rate $\geq 22 /$ min), hypotension (systolic blood pressure $\leq 100 \mathrm{~mm} \mathrm{Hg}$ ), and altered mental status (GCS <12)

qSOFA can promptly identify patients with suspected infection at high risk of longer ICU stay or hospital mortality

SOFA was a better predictor of hospital mortality than SIRS or qSOFA among ICU patients

qSOFA was a better predictor of hospital mortality among patients with suspected infection outside the ICU compared with SIRS and SOFA

Shankar-Hari et al.

Definition of septic shock: "subset of sepsis in which underlying circulatory, cellular, and metabolic

abnormalities are associated with a greater risk of mortality than sepsis alone"

New septic shock clinical criteria: hypotension requiring vasopressor(s) to maintain mean arterial pressure $\geq 65 \mathrm{~mm} \mathrm{Hg}$ AND serum lactate level $>2 \mathrm{mmol} / \mathrm{L}(18 \mathrm{mg} / \mathrm{dL})$ after adequate fluid resuscitation

Singer et al.

Definition of sepsis: "life-threatening organ dysfunction caused by a dysregulated host response to infection" Under the new sepsis definition, the term "severe sepsis" was considered redundant and has been removed SIRS criteria removed because of inadequate specificity and sensitivity for sepsis diagnosis

Identification of organ dysfunction: acute change in total SOFA score $\geq 2$ points consequent to the infection; in patients with unknown preexisting organ dysfunction, the baseline SOFA score can be assumed to be zero Hospital mortality in septic shock identified with these criteria $>40 \%$

\section{Strengths}

$[108,109]$

The new definitions developed by the analysis of large multicenter cohorts using quantitative, objective data The definitions were developed by an internationally recognized, multidisciplinary group of sepsis experts The updated diagnostic criteria for sepsis are broad and may assist evaluation of the epidemiology and economics related to sepsis qSOFA is a simple and rapid bedside score

\section{Concerns}

$[108,109]$

New sepsis definition may diagnose patients at a later stage of disease and with a worse prognosis in whom novel therapies are ineffective (as shown by Russell et al. [110] in the reanalysis of vasopressin in VASST) and do not screen patients who are potentially septic (and would benefit from early intervention)

SOFA may not be suitable in resource-limited settings

Requirement for serum lactate measurements

Definitions were derived from data from high-income countries, in particular, adults from the USA

The missing clinical data for the qSOFA score in patients outside ICUs demand prospective, real-world validation for use in clinical practice qSOFA score will identify sick patients who are not septic, i.e., may have inadequate specificity SIRS has been used as inclusion criterion in clinical trials and for the development of management bundles Molecular components of sepsis were not evaluated

GCS, Glasgow Coma Scale; ICU, intensive care unit; LODS, Logistic Organ Dysfunction Score; qSOFA, quick SOFA; SIRS, systemic inflammatory response syndrome; SOFA, Sequential Organ Failure Assessment.

optimized according to pharmacokinetic/pharmacodynamic principles, with no specific recommendation about the use of $\beta$-lactam CI.

Another RCT conducted in the Netherlands assessed the efficacy and safety of procalcitonin (PCT)-guided antibiotic treatment compared to usual antibiotic duration (i.e. without knowledge of PCT levels) in critically ill patients with an assumed or proven infection [40]. The PCT-guided intervention decreased the antibiotic consumption, lowered the duration of antibiotic treatment, and increased the number of antibiotic-free days, but there were no differences in ICU and hospital length of 
stay (LOS). Surprisingly, even thought this was a noninferiority trial in relation to 28-day and 1-year mortality, the intervention group had significantly lower (28-day and 1-year) mortality compared with usual care (even though the PCT-guided group had higher reinfection rates). The reason suggested by the authors for this discordance of reinfection rates and mortality is that perhaps physicians looked for an alternative diagnosis earlier if procalcitonin concentrations were low. Based on these results, a procalcitonin-guided strategy seems useful as a guide to antibiotic discontinuation. However, it still needs to be proven how cost-effective this strategy would be in real life.

Guidelines from SSC 2016 [17] suggest (as a weak recommendation with low quality of evidence) that procalcitonin levels can be used to support the discontinuation of empiric antibiotics in patients with sepsis who have clinical evidence of infection.

It is uncertain how to manage antifungals in patients at high risk of fungal infection in the ICU. Accordingly, the use of antifungal empirical therapy was evaluated in a multicenter RCT comparing micafungin empirical treatment with placebo in patients who had ICU-acquired sepsis, multiple Candida colonization, multiple organ failure, and exposure to broad-spectrum antibacterial agents [41]. The invasive fungal infection-free survival on day 28 (the primary outcome) was similar between the two groups, as were secondary outcomes and exploratory subgroup analyses. The single (but granted the primary outcome) significant positive finding was related to the number of invasive fungal infections at the 28-day follow-up, which was lower in the intervention group. Despite negative secondary findings, two important messages were highlighted by the authors. First, the occurrence of sepsis in patients with multiple organ dysfunction and multiple-site colonization on broad-spectrum antibiotics is infrequently due to invasive fungal infection. Second, the discrimination power of Candida colonization for invasive fungal infections may not be adequately precise to justify the costs of fungal culture systematic surveillance in ICU patients.

In general, for the management of infection, the SSC 2016 [17] recommends the administration of broad-spectrum intravenous antimicrobials for all likely pathogens (including bacterial and potentially fungal or viral coverage) within $1 \mathrm{~h}$ after sepsis recognition, and source control through a specific anatomic diagnosis of infection as rapidly as is practical. The Infectious Diseases Society of America (IDSA) [42] recommends the use of an echinocandin for suspected candidiasis in nonneutropenic ICU patients.

Update of Sepsis in the ICU

\section{Fluids}

Although fluid resuscitation is a crucial element of sepsis treatment (along with antimicrobials and vasopressors), how much fluid and the choice of the fluid to be utilized remain controversial, particularly after the negative results from 3 large RCTs of EGDT [43] compared with standard care [44-46].

A multicenter feasibility trial compared a conservative versus a liberal fluid approach after initial resuscitation in ICU patients with septic shock [47] and demonstrated that both the amount of resuscitation fluid in the first 5 days and during the entire ICU stay were lower in the conservative group. Based on this finding and that the conservative group had a lower frequency of worsening of renal function during the 90-day period (in comparison with the liberal group), the authors concluded that a conservative resuscitation protocol in ICU patients with septic shock is feasible and potentially beneficial. Major drawbacks of this trial were the impossibility of blinding and the differences in co-interventions between the two groups, which may have confounded the findings.

A systematic review and meta-analysis evaluated allcause mortality (at the latest time point available up to 90 days) of conservative versus deresuscitative fluid strategies in adults (and children) with ARDS, sepsis, or the systemic inflammatory response syndrome, in the postresuscitation phase of critical illness [48]. In contrast to several previous observational cohort studies $[49,50]$, this intervention trial found no associations between deresuscitative strategy and lower mortality rates or RRT incidence between the two groups. However, the conservative fluid approach was associated with greater ventilator-free days and decreased length of ICU stay.

Larger RCTs are necessary to evaluate benefits and risks of lower compared with higher volumes of resuscitation fluid, and to determine optimal fluid strategies in sepsis and septic shock.

The SSC 2016 guidelines [17] for fluid therapy recommend the administration of $30 \mathrm{~mL} / \mathrm{kg}$ of intravenous crystalloid within $3 \mathrm{~h}$, with additional fluid based on frequent reassessment; the application of a fluid challenge technique in which fluids should be continued as hemodynamic factors continues to improve with the use of crystalloids (balanced crystalloids or saline).

\section{Vasopressors}

Clinicians have a menu of several vasopressors (e.g., norepinephrine, dopamine, epinephrine, terlipressin, vasopressin, and phenylephrine) for resuscitation of septic

J Innate Immun 2017;9:441-455

DOI: $10.1159 / 000477419$ 
shock A recent, very comprehensive meta-analysis [51] evaluated the effects of different vasopressor drugs and regimens (alone or in combination) in patients admitted to ICU with hypotensive shock. Five vasopressors (dopamine, epinephrine, terlipressin, vasopressin, and phenylephrine) were compared with norepinephrine with respect to in-hospital, ICU, and 1-year mortality rates. There were no differences in all conducted analysis, including the comparison between norepinephrine versus dopamine, which was in disagreement with previous studies and trials [52-55]. The prior trial of norepinephrine versus dopamine [56] showed that there were greater risks with dopamine (i.e. arrhythmias) than norepinephrine but no difference in mortality in the septic shock subgroup.

Although it seems that no vasopressor is superior to others in decreasing mortality rates in sepsis, SSC 2016 [17] guidelines recommend norepinephrine as the firstchoice vasopressor, as this agent is associated with lower adverse events rates (e.g. arrhythmias) than dopamine. Additionally, the guidelines [17] suggest that vasopressin or epinephrine (aiming to increase mean arterial pressure), or vasopressin (aiming to decrease norepinephrine doses) can be used as additional drugs to norepinephrine, in particular because of the "relative vasopressin deficiency" present in patients with septic shock. Terlipressin and phenylephrine are neither recommended nor suggested due to the long-acting action of the former (even though it has similar effects of vasopressin) and the paucity of clinical studies with the latter [17].

\section{Albumin}

The colloid crystalloid controversy continues and especially the role of albumin for resuscitation and maintenance fluid in sepsis and septic shock. Albumin for fluid resuscitation in sepsis was not associated with decrease mortality rates in 2 RCTs $[57,58]$. However, the ALBIOS RCT [57] demonstrated that the addition of $20 \%$ albumin to crystalloids reached higher targeted mean arterial pressure within $6 \mathrm{~h}$ of administration and lowered fluid balance over the first 7 days. There was a suggestion of lower mortality in the subgroup of patients who had septic shock.

It appears that the administration of albumin in sepsis is neither associated with advantages nor harm in sepsis and septic shock, and there is still equipoise that needs more clarification. Based on that, the updated SSC guidelines [17] suggest the addition of albumin to crystalloids if substantial amounts of crystalloids are required for initial resuscitation.

\section{Corticosteroids}

The corticosteroid controversy also continues in severe sepsis and in septic shock. A randomized placebocontrolled trial compared early therapy with hydrocortisone (or placebo) for the prevention of progression to septic shock in patients with severe sepsis [59]. Hydrocortisone or placebo was administered over the first 11 days (bolus dose followed by weaning doses), and septic shock progression was determined within the first 14 days. Neither septic shock progression nor mortality rates (at 28 and 90 days, at ICU, and in hospital) differed between the two groups. An unexpected finding was that the hydrocortisone group had a lower frequency of delirium compared with the placebo group, which contradicts prior studies [60,61], raising questions concerning the concept of cortisone-induced delirium in critically ill patients.

The SSC 2016 guidelines [17] suggest the intravenous use of hydrocortisone $(200 \mathrm{mg} /$ day) only in patients with septic shock in whom fluid resuscitation in association with vasopressors was not sufficient to restore hemodynamic stability.

\section{Sepsis-Associated Organ Dysfunction Management}

\section{Lung Dysfunction}

Pulmonary dysfunction is extremely frequent in patients with sepsis and associated with increased mortality rates, particularly when ARDS is diagnosed.

\section{ARDS Epidemiology}

Recently, a large epidemiological study [62] demonstrated that ARDS is prevalent (10.4\% of all ICU admissions), underrecognized, and associated with high mortality rates. In this study, the recognition of ARDS at the time of fulfillment of ARDS criteria varied from 32.0 to $36.0 \%$, and roughly $50 \%$ of all patients with mild ARDS were recognized by clinicians. In addition, the study supported the predictive validity of the Berlin Definition: the greater the severity of ARDS, the greater was the ICU and hospital stay, the number of days of invasive ventilation, and the mortality. Factors independently associated with higher probability of clinician recognition of ARDS were higher nurse-to-patient ratios, higher physician-to-patient ratios, younger patient age, lower $\mathrm{PaO}_{2} / \mathrm{FIO}_{2}$ ratio, and the presence of pneumonia or pancreatitis.

\section{Statins and ARDS}

The use of statins in patients with ARDS (and sepsis) is still an area of uncertainty. Findings from observation-
446

J Innate Immun 2017;9:441-455

DOI: $10.1159 / 000477419$
Genga/Russell 
al studies differed from RCTs [63]: while statins were associated with decreased mortality [64] and delirium incidence [65] in the former, these findings have not been supported by the latter $[66,67]$. It was suggested that perhaps the use of statins might be beneficial for some specific subgroups of patients presenting with ARDS and/or sepsis, given the significant clinical and pathological heterogeneity associated with both disorders [68]. Indeed, distinct subphenotypes of ARDS were associated with distinct responses to different ventilator strategies [69]. Other questions still to be answered are: (1) How do differences in hydrophilic and lipophilic properties of different statins (e.g., simvastatin and rosuvastatin) impact their efficacy and safety in sepsis/ARDS [70]? (2) Are statins more appropriate for the prevention or for the treatment of sepsis-associated ARDS [68]? (3) What is the adequate statin and statin dose associated with better outcomes in sepsis/ARDS [71]?

\section{Oxygen Therapy}

Postextubation High-Flow Nasal Cannula. The association between the use of a postextubation high-flow nasal cannula and reintubation risks was evaluated in 2 RCTs in critically ill patients considered at low [72] and high risk for reintubation [73]. The first trial compared a high-flow nasal cannula with conventional oxygen therapy in low-risk patients, while the second compared the same approach with noninvasive ventilation (NIV), in high-risk patients (designed as a noninferiority trial). In both studies, the use of a postextubation high-flow nasal cannula was associated with better outcomes: significantly decreased risk for reintubation within $72 \mathrm{~h}$ in patients at low risk for reintubation [72], and noninferior to NIV at preventing reintubation and postextubation respiratory failure in patients at high risk for reintubation [73]. Although the latter was a noninferiority trial [73], it showed a higher frequency of respiratory failure in patients receiving NIV than in those receiving oxygen by high-flow nasal cannula.

NIV via Helmet. Intubation rates were compared between patients with ARDS receiving NIV via helmet versus NIV via face mask [74]. Interestingly, this study had an early termination at the first interim analysis because it reached the criteria for its predetermined efficacy: patients receiving NIV via helmet had much lower intubation rates than those who received NIV via face mask (18.2 vs. $61.5 \%$, respectively). Greater effectiveness in the delivery of high positive end-expiratory pressure levels as well as air leak prevention associated with the helmet's neck seal may explain these results. Even though the study

Update of Sepsis in the ICU demonstrated compelling findings, it was a single-center study and thus may have overestimated the effect size (due to early termination); therefore, it warrants replication in larger multicenter studies. The SSC 2016 recommendations [17] for patients mechanically ventilated with sepsis-related ARDS are a target tidal volume of 6 $\mathrm{mL} / \mathrm{kg}$ of predicted body weight and a plateau pressure $\leq 30 \mathrm{~cm} \mathrm{H}_{2} \mathrm{O}$. There is no recommendation for the use of NIV in patients with sepsis-induced ARDS.

Conservative Oxygen Supplementation Protocol. ICU mortality rates were analyzed in a conservative oxygen supplementation protocol versus a standard approach in a single-center RCT [75]. Out of all medical and surgical critically ill patients included, around $21 \%$ had septic shock. The two approaches differed from each other according to partial pressure of $\mathrm{O}_{2}\left(\mathrm{PaO}_{2}\right)$ and/or peripheral capillary oxygen saturation $\left(\mathrm{SpO}_{2}\right)$ targets, which was lower in the conservative group. Even though the study found encouraging results (11.6 vs. $20.2 \%$ mortality rates in conservative vs. standard groups, respectively), two concerns deserve attention. First, this was a single-center trial and, second, the trial was terminated early due to a low inclusion rate, ending with a sample size $27 \%$ lower than expected. Despite that, these findings reinforce the need for larger multicenter trials to examine the potential benefits of a conservative oxygen approach in critically ill septic patients.

\section{Cardiac Dysfunction}

Ventricular dysfunction is a common complication of sepsis and septic shock, and dobutamine is the most commonly used inotropic agent to correct ventricular dysfunction to date. Levosimendan increases ventricular contractility, is a mild vasodilator, and has anti-inflammatory properties in sepsis. Levosimendan was evaluated in a randomized placebo-controlled trial in adults with septic shock as an additional drug to standard treatment [76]. Median daily SOFA scores up to day 28 (the primary outcome) were similar in placebo and levosimendan groups, as well as 28-day, ICU, and hospital mortality. Additionally, patients who received levosimendan had greater rates of supraventricular tachyarrhythmia and a lower likelihood of successful weaning from mechanical ventilation than patients in the placebo group. Although levosimendan was not associated with overall less severe organ dysfunction or lower mortality, a limitation of this trial was that few patients had a low measured cardiac output and none had echocardiographic analyses. Additional trial(s) of levosimendan in patients who have a decreased cardiac output in septic shock are still warranted.

J Innate Immun 2017;9:441-455 DOI: $10.1159 / 000477419$ 
Table 2. Summary of AKIKI [77] and ELAIN trials [78]

\begin{tabular}{|c|c|c|}
\hline & AKIKI [77] & ELAIN [78] \\
\hline Study type & $\begin{array}{l}\text { Unblinded, prospective, multicenter, open-label, } \\
\text { 2-group RCT in } 31 \text { ICUs in France }\end{array}$ & Single-center, 2-group, parallel-group RCT in Germany \\
\hline Sample size & $\begin{array}{l}\text { Early strategy }(n=311) \\
\text { Delayed strategy }(n=308)\end{array}$ & $\begin{array}{l}\text { Early strategy }(n=112) \\
\text { Delayed strategy }(n=119)\end{array}$ \\
\hline $\begin{array}{l}\text { Eligibility } \\
\text { criteria }\end{array}$ & $\begin{array}{l}\text { Critically ill adults classified as KDIGO stage } 3 \\
\text { receiving invasive mechanical ventilation, } \\
\text { catecholamine infusion (epinephrine or } \\
\text { norepinephrine), or both. }\end{array}$ & $\begin{array}{l}\text { Critically ill patients with AKI KDIGO stage } 2 \text { and plasma } \\
\text { NGAL }>150 \mathrm{ng} / \mathrm{mL}\end{array}$ \\
\hline Early strategy & $\begin{array}{l}\text { RRT initiated } 6 \mathrm{~h} \text { after documentation of } \\
\text { KDIGO stage } 3\end{array}$ & RRT initiated within $8 \mathrm{~h}$ after diagnosis of KDIGO stage 2 \\
\hline $\begin{array}{l}\text { Delayed } \\
\text { strategy }\end{array}$ & $\begin{array}{l}\text { RRT initiated if abnormal potassium, urea, or } \mathrm{pH} \text {; } \\
\text { acute pulmonary edema; oliguria, or anuria } \\
\text { lasting }>72 \mathrm{~h} \text { after randomization }\end{array}$ & $\begin{array}{l}\text { RRT initiated within } 12 \mathrm{~h} \text { of stage } 3 \mathrm{AKI} \text {, or if abnormal } \\
\text { urea, potassium, or magnesium; oliguria or anuria; or } \\
\text { organ edema in the presence of AKI resistant to diuretic } \\
\text { treatment }\end{array}$ \\
\hline $\begin{array}{l}\text { Primary } \\
\text { outcome }\end{array}$ & Overall 60-day survival & Overall 90-day mortality \\
\hline $\begin{array}{l}\text { Main secondary } \\
\text { outcomes }\end{array}$ & $\begin{array}{l}\text { Days free of RRT, mechanical ventilation, or } \\
\text { vasopressors, day-3 and - } 7 \text { SOFA scores, ICU and } \\
\text { hospital LOS, nosocomial infections, } \\
\text { complications potentially related to AKI or RRT }\end{array}$ & $\begin{array}{l}\text { Duration of RRT, daily SOFA scores in ICU, recovery of } \\
\text { renal function, requirement of hemodialysis after } 28 \text { and } \\
60 \text { days; duration of renal support; ICU and hospital LOS }\end{array}$ \\
\hline Main results & $\begin{array}{l}\text { No difference in the primary outcome between } \\
\text { study groups } \\
\text { Early strategy group had fewer RRT-free days and } \\
\text { greater frequency of catheter-related bloodstream } \\
\text { infections compared to delayed strategy }\end{array}$ & $\begin{array}{l}\text { Early strategy decreased 90-day mortality and median } \\
\text { duration of RRT, improved rates of renal recovery on day } \\
\text { 90, and had shorter hospital LOS and duration of } \\
\text { mechanical ventilation compared with delayed strategy } \\
\text { No differences between groups in } 28 \text { - and } 60 \text {-day } \\
\text { mortality }\end{array}$ \\
\hline Comments & $\begin{array}{l}\text { Delayed RRT obviated need for RRT } \\
\text { The study included patients with advanced kidney } \\
\text { injury (stage } 3 \text { KDIGO), which limits its } \\
\text { generalizability }\end{array}$ & $\begin{array}{l}\text { Faster metabolic and/or uremic control, and more } \\
\text { effective prevention and/or management of fluid overload } \\
\text { were potential benefits associated with early RRT } \\
\text { approach } \\
\text { Use of NGAL in association with KDIGO classification } \\
\text { might have reduced the rates of "unnecessary" RRT in the } \\
\text { early strategy group } \\
\text { Possible overestimation of observed effects due to the } \\
\text { single-center study design }\end{array}$ \\
\hline
\end{tabular}

AKI, acute kidney injury; AKIKI, Artificial Kidney Initiation in Kidney Injury; ELAIN, Early versus Late Initiation of Renal Replacement Therapy in Critically Ill Patients with Acute Kidney Injury; ICU, intensive care unit; KDIGO, Kidney Disease Improving Global Outcomes; LOS, length of stay; NGAL, neutrophil gelatinase-associated lipocalin; RCT, randomized controlled trial; RRT, renal replacement therapy; SOFA, Sequential Organ Failure Assessment.

\section{Kidney Dysfunction and Acute Kidney Injury}

Although sepsis-associated acute kidney injury (AKI) is common, the optimal timing for the initiation of RRT is still unclear. Two recent RCTs (AKIKI [77] and ELAIN [78]) addressed this question by comparing early versus delayed initiation of RRT in critically ill patients and are described in Table 2. Although both RCTs analyzed dif- ferent populations regarding the severity of kidney injury, they demonstrated quite distinct results, supporting the necessity of larger RCTs in different geographic regions: while the latter found decreases in mortality rates when RRT was initiated early, no mortality difference between the two strategies was demonstrated by the latter.
Genga/Russell 
Table 3. Delirium incidence in critically ill patients: summary of a dynamic light application (DLA) study [88] and an ancillary study using statins for acutely injured lungs from sepsis (SAILS) [89]

\begin{tabular}{|c|c|c|}
\hline & DLA to prevent ICU-acquired delirium [88] & Ancillary study within the SAILS trial [89] \\
\hline Type of study & Single-center RCT & $\begin{array}{l}\text { Ancillary study within the SAILS trial (RCT } \\
\text { that assessed rosuvastatin vs. placebo for } \\
\text { mortality and ventilator-free days in } \\
\text { patients with sepsis-associated ARDS) }\end{array}$ \\
\hline Rationale & $\begin{array}{l}\text { Bright-light therapy might reduce } \\
\text { disturbances in the circadian rhythm and } \\
\text { sleep (associated with delirium incidence), } \\
\text { associated with decreased restless behavior } \\
\text { in geriatric patients with delirium and in } \\
\text { postoperative patients }\end{array}$ & $\begin{array}{l}\text { Long-term cognitive impairment involves } \\
\text { neuroinflammation } \\
\text { Statins have anti-inflammatory effects }\end{array}$ \\
\hline Sample size & $\begin{array}{l}\text { Intervention group }(n=354) \\
\text { Control group }(n=360)\end{array}$ & $\begin{array}{l}\text { Intervention group }(n=67) \\
\text { Placebo group }(n=82)\end{array}$ \\
\hline $\begin{array}{l}\text { Eligibility } \\
\text { criteria }\end{array}$ & Adult patients with expected ICU stay $>24 \mathrm{~h}$ & $\begin{array}{l}\text { Patients of SAILS RCT who had assessment } \\
\text { of delirium and cognitive outcomes }\end{array}$ \\
\hline Intervention & Dynamic lighting application $^{1}$ & Rosuvastatin \\
\hline $\begin{array}{l}\text { Primary } \\
\text { outcomes }\end{array}$ & $\begin{array}{l}\text { Cumulative incidence of ICU-acquired } \\
\text { delirium (CAM-ICU) }\end{array}$ & $\begin{array}{l}\text { Daily delirium status in ICU up to } 28 \text { days } \\
\text { (CAM-ICU) }\end{array}$ \\
\hline Main results & Delirium incidence similar between groups & Delirium status similar between groups \\
\hline Comments & $\begin{array}{l}\text { Light effects on biological clock may need } \\
\text { open eyes for retinal input through } \\
\text { photosensitive ganglion cells } \\
\text { Most patients were sedated with eyes closed } \\
\text { The normal circadian rhythm was affected by } \\
\text { sedation }\end{array}$ & $\begin{array}{l}\text { Major findings differ from prior } \\
\text { observational cohort studies of simvastatin } \\
\text { Included young patients with ARDS, so } \\
\text { cannot be generalized to all critically ill } \\
\text { patients } \\
\text { Simvastatin has greater brain penetration } \\
\text { than rosuvastatin }\end{array}$ \\
\hline
\end{tabular}

ARDS, acute respiratory distress syndrome; CAM-ICU, Confusion Assessment Method for the ICU; ICU, intensive care unit; RCT, randomized controlled trial.

${ }^{1}$ Application of lighting level and color temperature at specific time points during the day.

A very recent, large, factorial $(2 \times 2)$, double-blind RCT [79] compared kidney function - measured as the number of kidney failure-free days within 28 days - between patients with septic shock receiving vasopressin (plus hydrocortisone or placebo) or norepinephrine (plus hydrocortisone or placebo). The rationale derives from post hoc VASST [80] studies suggesting that use of vasopressin compared to norepinephrine alone might prevent worsening of renal failure [79], and from discovery of a potentially beneficial interaction between vasopressin and steroid treatment in septic shock $[81,82]$. Despite the use of higher doses of vasopressin ( $0.06 \mathrm{vs} .0 .03 \mathrm{U} / \mathrm{min}$ in VASST) [83], there was no difference between groups in the primary endpoint. However, vasopressin was associated with a significantly lower rate of RRT than norepinephrine.

Update of Sepsis in the ICU
The 3 recent RCTs of EGDT [44-46] found similar rates of AKI development and other AKI-related outcomes (AKI duration and RRT therapy) among patients treated with a protocol-based approach versus usual care. In addition, an ancillary study to the ProCESS trial analyzing AKI patients [84] further demonstrated that a protocolized resuscitation had no effect on renal function recovery (complete or partial) compared with usual care.

Based on these studies, the appropriate approach for the prevention and/or the management of kidney dysfunction associated with sepsis is still to be clarified. The SSC 2016 [17] guidelines present only weak recommendations regarding the type of RRT in patients with sepsis (either continuous or intermittent RRT are recommend-

J Innate Immun 2017;9:441-455 DOI: $10.1159 / 000477419$ 
ed), preferring the former in hemodynamically unstable septic patients to achieve a better management of fluid balance. No recommendation or suggestion regarding the timing for the initiation of RRT in sepsis was defined by these guidelines [17].

\section{Brain Dysfunction}

Delirium is common among critically ill - especially septic - patients [85] and is associated with poor shortand long-term outcomes (e.g., increased mortality rates [86] and impaired cognitive function [87]). Delirium incidence was evaluated in 2 recent RCTs using dynamic light application [88] and rosuvastatin [89], described in Table 3.

\section{Dexmedetomidine}

The use of dexmedetomidine was supported by Reade et al. [90] in a placebo-controlled trial that analyzed a very specific group of critically ill patients - those with delirium receiving mechanical ventilation in whom extubation was considered inappropriate because of the severity of agitation and delirium. Patients treated with dexmedetomidine had greater number of ventilator-free hours in the 7 days after randomization (primary outcome) than the placebo-treated group. Despite this finding, two comments are important to mention. Although clinicians were blinded to study drug allocation, the frequent dexmedetomidine-associated bradycardia may have suggested patients' allocation and, second, the benefits of the study drug cannot be generalized to patients in the early phases of their critical illness and/or who are not intubated.

The role of dexmedetomidine for the prevention/ treatment of delirium needs further research, and no specific recommendation with respect to delirium prevention or treatment was presented in the SSC 2016 guidelines [17]. The Pain, Agitation, and Delirium (PAD) 2013 guidelines [91] recommend routine monitoring for delirium in adult ICU patients using The Confusion Assessment Method for the ICU (CAM-ICU) and the Intensive Care Delirium Screening Checklist (ICDSC), early mobilization for reduction of the incidence and the duration of delirium, but no specific drug for delirium prevention. The same guidelines [91] suggest the use of dexmedetomidine as intravenous $\mathrm{CI}$ over the use of benzodiazepines in adult ICU patients with delirium unrelated to alcohol or benzodiazepine withdrawal to reduce the duration of delirium.

\section{Red Blood Cell Transfusion}

The UK National Clinical Guideline Centre's 2015 guidelines on transfusion of red blood cells (RBC) [92] were published by Carson et al. [93]. The two major recommendations (both strong with moderate quality of evidence) - based on a thorough systematic review of studies in critically ill patients - are: (1) hemodynamically stable patients should be managed according to a restrictive $\mathrm{RBC}$ transfusion approach, in which the threshold for transfusion is a hemoglobin level $<7 \mathrm{~g} / \mathrm{dL}$ (rather than a liberal approach [threshold of hemoglobin $<10 \mathrm{~g} / \mathrm{dL}]$ ), and (2) the age of the RBC units to be transfused should be the standard, i.e. the units can be selected at any point within their licensed dating period (i.e., no recommendation for the use of fresh units, defined as those with less than 10 days of processing).

The safety of a restrictive RBC transfusion approach (in comparison to a liberal approach) in patients with septic shock was first demonstrated by Holst et al. [94]. The two groups had no differences in mortality rates within 90 days (primary outcome) and all secondary outcomes including vasopressor or inotropic agent use, RRT, invasive or noninvasive mechanical ventilation, and ischemic event rates.

A recent meta-analysis regarding restrictive versus liberal transfusion strategies in sepsis confirmed the safety of the restrictive strategy regarding mortality (in hospital and at 28 and 90 days) [95]. RBC transfusion was associated with greater rates of nosocomial infections, acute lung injury, and AKI. Two major limitations from this study are that the meta-analysis was conducted only with cohort studies, and there was a high heterogeneity between the studies analyzed.

Based on these recent publications [92, 93, 95], 2 relevant RCTs [94, 96], and the SSC 2016 guidelines [17], the use of the restrictive strategy for stable patients with sepsis is recommended because it is safe, and the age of RBCs does not affect mortality.

\section{Nutrition}

Harvey et al. [97] showed no differences in 30-day mortality rates between critically ill patients receiving early enteral nutrition versus early parenteral nutrition. This finding was replicated in a recent multicenter RCT that included an integrated economic evaluation conducted in the UK [98]. The additional finding from this study was that parenteral nutrition had greater costs than
Genga/Russell 
Fig. 1. New targets and novel randomized controlled trial (RCT) designs are necessary in sepsis. The increased number of sepsis survivors and subsequent greater proportions and numbers of sepsis-associated long-term outcomes, and a great number of negative RCTs in sepsis emphasize the need for the use of new designs in RCTs (e.g., more and larger phase 2 trials, inclusion of patients at early stages of sepsis, and use of long-term and composite endpoints) and new targets aimed at immune system modulation, resolution of organ dysfunction(s), and/or increased pathogen clearance.

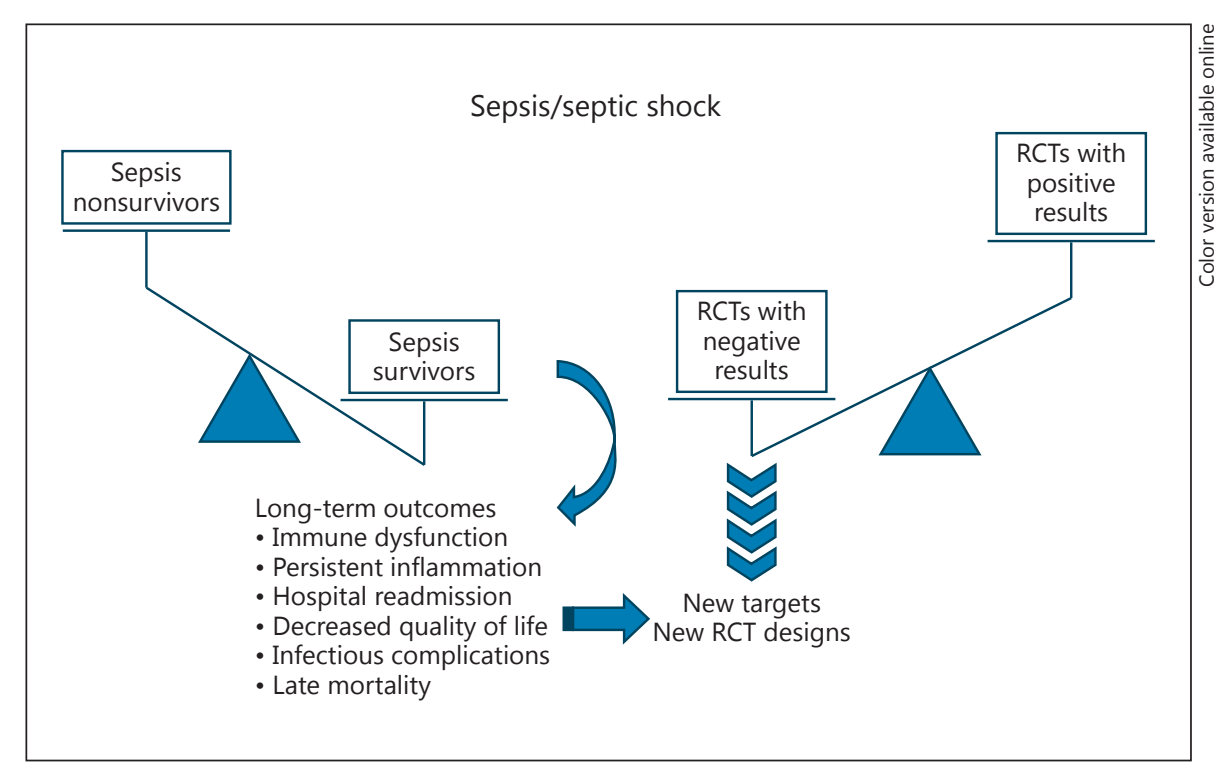

the enteral route resulting in a negative incremental net balance at 1 year.

Among other nutritional recommendations, the SSC 2016 guidelines [17] recommend against parenteral nutrition (alone or in combination with enteral feeding) in patients with sepsis or septic shock within the first 7 days. SSC also recommends early initiation of enteral nutrition in patients who can be fed, and intravenous glucose with advanced enteral feeds (e.g., increases in enteral diet infusion of $25 \mathrm{~mL} / \mathrm{h}$ every $4-8 \mathrm{~h}$ for gastric tubes or $6-12 \mathrm{~h}$ for duodenal tubes) as tolerated for patients in whom enteral feeding is not feasible over the first 7 days.

\section{Novel Targets for Sepsis}

Based on the relevance of immune system dysfunction in sepsis, interventions targeted at immunomodulation have been studied in sepsis, such as use of granulocyte and granulocyte-macrophage colony stimulating factor, interferon- $\gamma$ ) programmed cell death protein (PD)- 1 and $\mathrm{PD}$ ligands (PD-L1), recombinant human interleukin (IL)-3, IL-7, and IL-15, propranolol, oxandrolone, and dronabinol [29]. IL-7 (NCT02640807) and anti PD-L1 (NCT02576457) are currently being tested in clinical trials. Major findings associated with these therapies in sepsis include effective restoration of monocytic immunocompetence and potential reduction in mechanical ventilator days and other organ dysfunction with the use of granulocyte-macrophage colony stimulating factor [99]

Update of Sepsis in the ICU and decreased nosocomial infections with the inhibition of PD-1 and PD-L1 pathways in an animal model of sepsis [100].

Another attractive candidate for sepsis treatment is PCSK9 (proprotein convertase subtilisin/kexin type-9). Normal clearance of pathogen lipids such as lipopolysaccharide and lipoteichoic acid occurs via the hepatic lowdensity lipoprotein (LDL) receptor pathway, particularly on hepatocytes [101]. Pathogen lipids are carried within lipoprotein particles (high-density lipoprotein [HDL], very low-density lipoprotein and LDL-cholesterol) and transferred between these fractions especially from HDL to LDL. Transfer proteins such as phospholipid transfer protein and lipopolysaccharide binding protein guide this process [102]. PCSK9 increases LDL receptor activity by hepatocyte lysosomal degradation and thus decreases pathogen lipid clearance [102]. Subsequently, PCSK9 knockout and PCSK9 inhibition decrease mortality and markers of inflammation such as plasma cytokines. Furthermore, plasma PCSK9 levels are increased in sepsis and correlated with the development of subsequent cardiovascular and respiratory failure [103]. Additionally, anti-PCSK9 therapy is associated with increased pathogen clearance and decreased inflammatory responses in a murine model of sepsis [104]. The pharmacological inhibition of PCSK9 results in greater availability of LDL receptors for pathogen clearance and may improve outcome in patients with sepsis.

J Innate Immun 2017;9:441-455 DOI: $10.1159 / 000477419$ 


\section{The Need for New Targets and Designs of RCTs in Sepsis}

Unfortunately, most pivotal RCTs in sepsis have been associated with negative results and no novel therapies have been introduced into clinical practice over the last years. In addition, the uncertainty about the best approach for hemodynamic therapy in sepsis has increased, particularly after the negative three "post-Rivers" EGDT trials [105]. Research regarding new targets in sepsis are necessary, such as therapies aiming at the improved organ dysfunction resolution (e.g. lipoxin A4) [106] and/or increased pathogen clearance (e.g. anti-PCSK9) [102]. Sepsis is a very heterogeneous condition and, accordingly, better selection of responsive patients early in the course of sepsis may improve the chances of finding a new and effective treatment [106]. Proof-of-principle phase 2 RCTs have been positive but then negative phase III pivotal RCTs followed, showing that phase II RCs have been "false positives" of most therapies. We suggest that phase II RCTs (1) should be conducted in larger patient cohorts and (2) should be targeted at the discovery of novel predictive biomarkers (i.e., defining increased response to therapy). Another change in pivotal RCTs is to move away from mortality because mortality has decreased and has been shown too insensitive to acute interventions. Some RCTs are now using organ dysfunction, such as more days alive and free of vasopressors and ventilation, as a novel primary composite endpoint. The field is also increasing interest in outcomes, i.e. even beyond 90-day mortality time points (Fig. 1). Finally, the production of secondary publications originated from large RCTs should be encouraged especially in sepsis through responsible data sharing to increase opportunities for further scientific and hypothesis-generating discoveries [107].

\section{Conclusions}

Sepsis mortality has decreased with concurrent increases in the proportions and numbers of sepsis survivors, and, consequently, sepsis-associated long-term outcomes have attracted increasing attention. Although an increasing number of studies focus on these long-term complications of sepsis we do need a much better understanding of its epidemiology, pathogenesis, management, and prevention. Immune system dysfunction both during and after sepsis may decrease late mortality and hospital readmissions. Despite the development of new sepsis definitions and updated sepsis guidelines, more RCTs possibly using new (1) targets, designs, and endpoints and (2) predictive biomarkers are fundamental to discover effective therapies that improve sepsis and septic shock outcomes.

\section{Disclosure Statement}

Dr. Genga has no conflicts of interest.

Dr. Russell reports patents owned by the University of British Columbia (UBC) that are related to PCSK9 inhibitor(s) and sepsis and related to the use of vasopressin in septic shock. Dr. Russell is an inventor on these patents. He is a founder, director, and shareholder in Cyon Therapeutics Inc. (developing a sepsis therapy) and has share options in Leading Biosciences Inc. He is a shareholder in Molecular You Corp. Dr. Russell reports receiving consulting fees from: Cubist Pharmaceuticals (now owned by Merck [formerly Trius Pharmaceuticals] developing antibiotics), Leading Biosciences (developing a sepsis therapeutic), Ferring Pharmaceuticals (manufactures vasopressin and is developing selepressin), Grifols (sells albumin), La Jolla Pharmaceuticals (developing angiotensin II; Dr. Russell chairs the DSMB of a trial of angiotensin II), CytoVale Inc. (developing a sepsis diagnostic), and Asahi Kesai Pharmaceuticals of America (AKPA) (developing recombinant thrombomodulin). He reports having received an investigator-initiated grant from Grifols that is provided to and administered by UBC.

\section{References}

1 Hall MJ, Williams SN, DeFrances CJ, Golosinskiy A: Inpatient care for septicemia or sepsis: a challenge for patients and hospitals. NCHS Data Brief 2011;62:1-8.

2 Hoyert DL, Xu J: Deaths: preliminary data for 2011. Natl Vital Stat Rep 2012;61:1-51.

3 Navaneelan T, Alam S, Peters PA, Phillips O: Deaths involving sepsis in Canada. Health at a Glance. 2016, http://www.statcan.gc.ca/ pub/82-624-x/2016001/article/14308-eng. htm.
4 Perner A, Gordon AC, De Backer D, et al: Sepsis: frontiers in diagnosis, resuscitation and antibiotic therapy. Intensive Care Med 2016; 42:1958-1969.

5 Chang DW, Tseng CH, Shapiro MF: Rehospitalizations following sepsis: common and costly. Crit Care Med 2015;43:2085-2093.

6 Donnelly JP, Hohmann SF, Wang HE: Unplanned readmissions after hospitalization for severe sepsis at academic medical centeraffiliated hospitals. Crit Care Med 2015;43: 1916-1927.
7 Goodwin AJ, Rice DA, Simpson KN, Ford DW: Frequency, cost, and risk factors of readmissions among severe sepsis survivors. Crit Care Med 2015;43:738-746.

8 Jones TK, Fuchs BD, Small DS, et al: Postacute care use and hospital readmission after sepsis. Ann Am Thorac Soc 2015;12:904-913.

9 Liu V, Lei X, Prescott HC, et al: Hospital readmission and healthcare utilization following sepsis in community settings. J Hosp Med 2014;9:502-507. 
10 Mayr FB, Talisa VB, Balakumar V, et al: Proportion and cost of unplanned 30-day readmissions after sepsis compared with other medical conditions. JAMA 2017;317:530531.

11 Ortego A, Gaieski DF, Fuchs BD, et al: Hospital-based acute care use in survivors of septic shock. Crit Care Med 2015;43:729-737.

12 Prescott HC, Langa KM, Liu V, Escobar GJ, Iwashyna TJ: Increased 1-year healthcare use in survivors of severe sepsis. Am J Respir Crit Care Med 2014;190:62-69.

13 Kissoon N, Daniels R, van der Poll T, Finfer S, Reinhart K: Sepsis - the final common pathway to death from multiple organ failure in infection. Crit Care Med 2016;44:e446.

14 Wang H, Naghavi M, Allen C: Global, regional, and national life expectancy, all-cause mortality, and cause-specific mortality for 249 causes of death, 1980-2015: a systematic analysis for the Global Burden of Disease Study 2015. Lancet 2016;388:1459-1544.

15 Fleischmann C, Scherag A, Adhikari NK, et al: Assessment of global incidence and mortality of hospital-treated sepsis. Current estimates and limitations. Am J Respir Crit Care Med 2016;193:259-272.

16 Rhee C, Gohil S, Klompas M: Regulatory mandates for sepsis care - reasons for caution. N Engl J Med 2014;370:1673-1676.

17 Rhodes A, Evans LE, Alhazzani W, et al: Surviving Sepsis Campaign: International Guidelines for Management of Sepsis and Septic Shock: 2016. Crit Care Med 2017;45:486-552.

18 Gaieski DF, Edwards JM, Kallan MJ, Carr BG: Benchmarking the incidence and mortality of severe sepsis in the United States. Crit Care Med 2013;41:1167-1174.

19 Kaukonen KM, Bailey M, Suzuki S, Pilcher D, Bellomo R: Mortality related to severe sepsis and septic shock among critically ill patients in Australia and New Zealand, 2000-2012. JAMA 2014;311:1308-1316.

20 Marik PE, Linde-Zwirble WT, Bittner EA, Sahatjian J, Hansell D: Fluid administration in severe sepsis and septic shock, patterns and outcomes: an analysis of a large national database. Intensive Care Med 2017;43:625-632.

21 Iwashyna TJ, Ely EW, Smith DM, Langa KM: Long-term cognitive impairment and functional disability among survivors of severe sepsis. JAMA 2010;304:1787-1794.

22 Jackson JC, Pandharipande PP, Girard TD, et al: Depression, post-traumatic stress disorder, and functional disability in survivors of critical illness in the BRAIN-ICU study: a longitudinal cohort study. Lancet Respir Med 2014; 2:369-379.

23 Winters BD, Eberlein M, Leung J, et al: Longterm mortality and quality of life in sepsis: a systematic review. Crit Care Med 2010;38: 1276-1283.

24 Sun A, Netzer G, Small DS, et al: Association between index hospitalization and hospital readmission in sepsis survivors. Crit Care Med 2016;44:478-487.
25 Linder A, Fjell C, Levin A, et al: Small acute increases in serum creatinine are associated with decreased long-term survival in the critically ill. Am J Respir Crit Care Med 2014;189: 1075-1081.

26 Schmidt K, Worrack S, Von Korff M, et al: Effect of a primary care management intervention on mental health-related quality of life among survivors of sepsis: a randomized clinical trial. JAMA 2016;315:2703-2711.

27 Goddard SL, Adhikari NK: The challenging task of improving the recovery of ICU survivors. JAMA 2016;315:2671-2672.

28 Yende S, D'Angelo G, Kellum JA, et al: Inflammatory markers at hospital discharge predict subsequent mortality after pneumonia and sepsis. Am J Respir Crit Care Med 2008;177:1242-1247.

29 Delano MJ, Ward PA: The immune system's role in sepsis progression, resolution, and long-term outcome. Immunol Rev 2016;274: 330-353.

30 Hotchkiss RS, Monneret G, Payen D: Sepsisinduced immunosuppression: from cellular dysfunctions to immunotherapy. Nat Rev Immunol 2013;13:862-874.

31 van Vught LA, Klein Klouwenberg PM, Spitoni C, et al: Incidence, risk factors, and attributable mortality of secondary infections in the intensive care unit after admission for sepsis. JAMA 2016;315:1469-1479.

32 Singer M, Deutschman CS, Seymour CW, et al: The Third International Consensus Definitions for Sepsis and Septic Shock (Sepsis-3). JAMA 2016;315:801-810.

33 American College of Chest Physicians/Society of Critical Care Medicine Consensus Conference: definitions for sepsis and organ failure and guidelines for the use of innovative therapies in sepsis. Crit Care Med 1992;20:864-874.

34 Levy MM, Fink MP, Marshall JC, et al: 2001 SCCM/ESICM/ACCP/ATS/SIS International Sepsis Definitions Conference. Crit Care Med 2003;31:1250-1256.

35 Seymour CW, Liu VX, Iwashyna TJ, et al: Assessment of clinical criteria for sepsis: for the Third International Consensus Definitions for Sepsis and Septic Shock (Sepsis-3). JAMA 2016:315:762-774.

36 Shankar-Hari M, Phillips GS, Levy ML, et al: Developing a new definition and assessing new clinical criteria for septic shock: for the Third International Consensus Definitions for Sepsis and Septic Shock (Sepsis-3). JAMA 2016;315:775-787.

37 Abdul-Aziz MH, Sulaiman H, Mat-Nor MB, et al: Beta-Lactam Infusion in Severe Sepsis (BLISS): a prospective, two-centre, open-labelled randomised controlled trial of continuous versus intermittent beta-lactam infusion in critically ill patients with severe sepsis. Intensive Care Med 2016;42:1535-1545.

38 Chytra I, Stepan M, Benes J, et al: Clinical and microbiological efficacy of continuous versus intermittent application of meropenem in critically ill patients: a randomized open-label controlled trial. Crit Care 2012;16:R113.
39 Dulhunty JM, Roberts JA, Davis JS, et al: A multicenter randomized trial of continuous versus intermittent $\beta$-lactam infusion in severe sepsis. Am J Respir Crit Care Med 2015; 192:1298-1305.

40 de Jong E, van Oers JA, Beishuizen A, et al: Efficacy and safety of procalcitonin guidance in reducing the duration of antibiotic treatment in critically ill patients: a randomised, controlled, open-label trial. Lancet Infect Dis 2016;16:819-827.

41 Timsit JF, Azoulay E, Schwebel C, et al: Empirical micafungin treatment and survival without invasive fungal infection in adults with ICU-acquired sepsis, Candida colonization, and multiple organ failure: the EMPIRICUS randomized clinical trial. JAMA 2016;316:1555-1564.

42 Pappas PG, Kauffman CA, Andes DR, et al: Clinical Practice Guideline for the Management of Candidiasis: 2016 Update by the Infectious Diseases Society of America. Clin Infect Dis 2016;62:e1-e50.

43 Rivers E, Nguyen B, Havstad S, et al: Early goal-directed therapy in the treatment of severe sepsis and septic shock. N Engl J Med 2001;345:1368-1377.

44 ARISE Investigators; ANZICS Clinical Trials Group, Peake SL, et al: Goal-directed resuscitation for patients with early septic shock. $\mathrm{N}$ Engl J Med 2014;371:1496-1506.

45 Mouncey PR, Osborn TM, Power GS, et al: Trial of early, goal-directed resuscitation for septic shock. N Engl J Med 2015;372:13011311.

46 Pro CI, Yealy DM, Kellum JA, et al: A randomized trial of protocol-based care for early septic shock. N Engl J Med 2014;370:16831693.

47 Hjortrup PB, Haase N, Bundgaard H, et al: Restricting volumes of resuscitation fluid in adults with septic shock after initial management: the CLASSIC randomised, parallelgroup, multicentre feasibility trial. Intensive Care Med 2016;42:1695-1705.

48 Silversides JA, Major E, Ferguson AJ, et al: Conservative fluid management or deresuscitation for patients with sepsis or acute respiratory distress syndrome following the resuscitation phase of critical illness: a systematic review and meta-analysis. Intensive Care Med 2017:43:155-170.

49 Boyd JH, Forbes J, Nakada TA, Walley KR, Russell JA: Fluid resuscitation in septic shock: a positive fluid balance and elevated central venous pressure are associated with increased mortality. Crit Care Med 2011;39:259-265.

50 Liu KD, Thompson BT, Ancukiewicz M, et al: Acute kidney injury in patients with acute lung injury: impact of fluid accumulation on classification of acute kidney injury and associated outcomes. Crit Care Med 2011;39: 2665-2671.

51 Gamper G, Havel C, Arrich J, et al: Vasopressors for hypotensive shock. Cochrane Database Syst Rev 2016;2:CD003709. 
52 Avni T, Lador A, Lev S, et al: Vasopressors for the treatment of septic shock: systematic review and meta-analysis. PLoS One 2015; 10:e0129305.

53 De Backer D, Aldecoa C, Njimi H, Vincent JL: Dopamine versus norepinephrine in the treatment of septic shock: a meta-analysis*. Crit Care Med 2012;40:725-730.

54 Sakr Y, Reinhart K, Vincent JL, et al: Does dopamine administration in shock influence outcome? Results of the Sepsis Occurrence in Acutely Ill Patients (SOAP) Study. Crit Care Med 2006;34:589-597.

55 Zhou F, Mao Z, Zeng X, et al: Vasopressors in septic shock: a systematic review and network meta-analysis. Ther Clin Risk Manag 2015;11: 1047-1059.

56 De Backer D, Biston P, Devriendt J, et al: Comparison of dopamine and norepinephrine in the treatment of shock. N Engl J Med 2010;362:779-789.

57 Caironi P, Tognoni G, Masson S, et al: Albumin replacement in patients with severe sepsis or septic shock. N Engl J Med 2014;370: 1412-1421.

58 Charpentier J, Mira JP: Efficacy and tolerance of hyperoncotic albumin administration in septic shock patients: the EARSS study. Intensive Care Med 2011;37(suppl 2):S115.

59 Keh D, Trips E, Marx G, et al: Effect of hydrocortisone on development of shock among patients with severe sepsis: the HYPRESS randomized clinical trial. JAMA 2016;316:17751785.

60 Nguyen DN, Huyghens L, Zhang H, et al: Cortisol is an associated-risk factor of brain dysfunction in patients with severe sepsis and septic shock. BioMed Res Int 2014;2014: 712742 .

61 Schreiber MP, Colantuoni E, Bienvenu OJ, et al: Corticosteroids and transition to delirium in patients with acute lung injury. Crit Care Med 2014;42:1480-1486.

62 Bellani G, Laffey JG, Pham T, et al: Epidemiology, patterns of care, and mortality for patients with acute respiratory distress syndrome in intensive care units in 50 countries. JAMA 2016;315:788-800.

63 Alhazzani W, Truwit J: Statins in patients with sepsis and ARDS: is it over? Yes. Intensive Care Med 2017;43:672-674.

64 Pasin L, Landoni G, Castro ML, et al: The effect of statins on mortality in septic patients: a meta-analysis of randomized controlled trials. PLoS One 2013;8:e82775.

65 Morandi A, Hughes CG, Thompson JL, et al: Statins and delirium during critical illness: a multicenter, prospective cohort study. Crit Care Med 2014;42:1899-1909.

66 McAuley DF, Laffey JG, O'Kane CM, et al: Simvastatin in the acute respiratory distress syndrome. N Engl J Med 2014;371:1695-1703.

67 National Heart, Lung, and Blood Institute ARDS Clinical Trials Network,, Truwit JD, Bernard GR, et al: Rosuvastatin for sepsis-associated acute respiratory distress syndrome. N Engl J Med 2014;370:2191-2200.
68 Kruger PS, Terblanche M: Statins in patients with sepsis and ARDS: is it over? No. Intensive Care Med 2017;43:675-676.

69 Calfee CS, Delucchi K, Parsons PE, et al: Subphenotypes in acute respiratory distress syndrome: latent class analysis of data from two randomised controlled trials. Lancet Respir Med 2014;2:611-620.

70 Morel J, Singer M: Statins, fibrates, thiazolidinediones and resveratrol as adjunctive therapies in sepsis: could mitochondria be a common target? Intensive Care Med Exp 2014;2: 9

71 McAuley D, Charles PE, Papazian L: Statins in patients with sepsis and ARDS: is it over? We are not sure. Intensive Care Med 2017;43: 677-679.

72 Hernandez G, Vaquero C, Gonzalez P, et al: Effect of postextubation high-flow nasal cannula vs conventional oxygen therapy on reintubation in low-risk patients: a randomized clinical trial. JAMA 2016;315:1354-1361.

73 Hernandez G, Vaquero C, Colinas L, et al: Effect of postextubation high-flow nasal cannula vs noninvasive ventilation on reintubation and postextubation respiratory failure in high-risk patients: a randomized clinical trial. JAMA 2016;316:1565-1574.

74 Patel BK, Wolfe KS, Pohlman AS, Hall JB, Kress JP: Effect of noninvasive ventilation delivered by helmet vs face mask on the rate of endotracheal intubation in patients with acute respiratory distress syndrome: a randomized clinical trial. JAMA 2016;315:24352441.

75 Girardis M, Busani S, Damiani E, et al: Effect of conservative vs conventional oxygen therapy on mortality among patients in an intensive care unit: the Oxygen-ICU Randomized Clinical Trial. JAMA 2016;316:1583-1589.

76 Gordon AC, Perkins GD, Singer M, et al: Levosimendan for the prevention of acute organ dysfunction in sepsis. N Engl J Med 2016;375: 1638-1648.

77 Gaudry S, Hajage D, Schortgen F, et al: Initiation strategies for renal-replacement therapy in the intensive care unit. N Engl J Med 2016; 375:122-133.

78 Zarbock A, Kellum JA, Schmidt C, et al: Effect of early vs delayed initiation of renal replacement therapy on mortality in critically ill patients with acute kidney injury: the ELAIN Randomized Clinical Trial. JAMA 2016;315: 2190-2199.

79 Gordon AC, Mason AJ, Thirunavukkarasu N, et al: Effect of early vasopressin vs norepinephrine on kidney failure in patients with septic shock: the VANISH Randomized Clinical Trial. JAMA 2016;316:509-518.

80 Russell JA, Walley KR, Singer J, et al: Vasopressin versus norepinephrine infusion in patients with septic shock. N Engl J Med 2008; 358:877-887.

81 Russell JA, Walley KR, Gordon AC, et al: Interaction of vasopressin infusion, corticosteroid treatment, and mortality of septic shock. Crit Care Med 2009;37:811-818.
82 Gordon AC, Mason AJ, Perkins GD, et al: The interaction of vasopressin and corticosteroids in septic shock: a pilot randomized controlled trial. Crit Care Med 2014;42:1325-1333.

83 Torgersen C, Dunser MW, Wenzel V, et al: Comparing two different arginine vasopressin doses in advanced vasodilatory shock: a randomized, controlled, open-label trial. Intensive Care Med 2010;36:57-65.

84 Kellum JA, Chawla LS, Keener C, et al: The effects of alternative resuscitation strategies on acute kidney injury in patients with septic shock. Am J Respir Crit Care Med 2016;193: 281-287.

85 Ely EW, Girard TD, Shintani AK, et al: Apolipoprotein E4 polymorphism as a genetic predisposition to delirium in critically ill patients. Crit Care Med 2007;35:112-117.

86 Ely EW, Shintani A, Truman B, et al: Delirium as a predictor of mortality in mechanically ventilated patients in the intensive care unit. JAMA 2004;291:1753-1762.

87 Pandharipande PP, Girard TD, Jackson JC, et al: Long-term cognitive impairment after critical illness. N Engl J Med 2013;369:1306-1316.

88 Simons KS, Laheij RJ, van den Boogaard M, et al: Dynamic light application therapy to reduce the incidence and duration of delirium in intensive-care patients: a randomised controlled trial. Lancet Respir Med 2016;4:194202.

89 Needham DM, Colantuoni E, Dinglas VD, et al: Rosuvastatin versus placebo for delirium in intensive care and subsequent cognitive impairment in patients with sepsis-associated acute respiratory distress syndrome: an ancillary study to a randomised controlled trial. Lancet Respir Med 2016;4:203-212.

90 Reade MC, Eastwood GM, Bellomo R, et al: Effect of dexmedetomidine added to standard care on ventilator-free time in patients with agitated delirium: a randomized clinical trial. JAMA 2016;315:1460-1468.

91 Barr J, Fraser GL, Puntillo K, et al: Clinical practice guidelines for the management of pain, agitation, and delirium in adult patients in the intensive care unit. Crit Care Med 2013; 41:263-306.

92 Padhi S, Kemmis-Betty S, Rajesh S, et al: Blood transfusion: summary of NICE guidance. BMJ 2015;351:h5832.

93 Carson JL, Guyatt G, Heddle NM, et al: Clinical practice guidelines from the AABB: red blood cell transfusion thresholds and storage. JAMA 2016;316:2025-2035.

94 Holst LB, Haase N, Wetterslev J, et al: Lower versus higher hemoglobin threshold for transfusion in septic shock. N Engl J Med 2014;371:1381-1391.

95 Dupuis C, Sonneville R, Adrie C, et al: Impact of transfusion on patients with sepsis admitted in intensive care unit: a systematic review and meta-analysis. Ann Intensive Care 2017; $7: 5$.

96 Lacroix J, Hebert PC, Fergusson DA, et al: Age of transfused blood in critically ill adults. N Engl J Med 2015;372:1410-1418. 
97 Harvey SE, Parrott F, Harrison DA, et al: Trial of the route of early nutritional support in critically ill adults. N Engl J Med 2014;371: 1673-1684.

98 Harvey SE, Parrott F, Harrison DA, et al: A multicentre, randomised controlled trial comparing the clinical effectiveness and cost-effectiveness of early nutritional support via the parenteral versus the enteral route in critically ill patients (CALORIES) Health Technol Assess 2016;20:1-144.

99 Meisel C, Schefold JC, Pschowski R, et al: Granulocyte-macrophage colony-stimulating factor to reverse sepsis-associated immunosuppression: a double-blind, randomized, placebo-controlled multicenter trial. Am J Respir Crit Care Med 2009;180:640648.

100 Chang KC, Burnham CA, Compton SM, et al: Blockade of the negative co-stimulatory molecules PD- 1 and CTLA-4 improves survival in primary and secondary fungal sepsis. Crit Care 2013;17:R85.
101 Topchiy E, Cirstea M, Kong HJ, et al: Lipopolysaccharide is cleared from the circulation by hepatocytes via the low density lipoprotein receptor. PLoS One 2016;11: e0155030.

102 Walley KR, Francis GA, Opal SM, et al: The central role of proprotein convertase subtilisin/kexin type 9 in septic pathogen lipid transport and clearance. Am J Respir Crit Care Med 2015;192:1275-1286.

103 Boyd JH, Fjell CD, Russell JA, et al: Increased plasma PCSK9 levels are associated with reduced endotoxin clearance and the development of acute organ failures during sepsis. J Innate Immun 2016;8:211-220.

104 Walley KR, Thain KR, Russell JA, et al: PCSK9 is a critical regulator of the innate immune response and septic shock outcome. Sci Transl Med 2014;6:258ra143.
105 Howell MD, Davis AM: Management of sepsis and septic shock. JAMA 2017;317:847848.

106 Matthay MA, Liu KD: New strategies for effective therapeutics in critically ill patients. JAMA 2016;315:747-748.

107 Ramanan M, Myburgh J, Finfer S, Bellomo R, Venkatesh B: Publication of secondary analyses from randomized trials in critical care. N Engl J Med 2016;375:2105-2106.

108 Abraham E: New definitions for sepsis and septic shock: continuing evolution but with much still to be done. JAMA 2016;315:757759.

109 Sprung CL, Schein RM, Balk RA: The new sepsis consensus definitions: the good, the bad and the ugly. Intensive Care Med 2016; 42:2024-2026.

110 Russell JA, Lee T, Singer J, et al: The Septic Shock 3.0 Definition and Trials: a vasopressin and septic shock trial experience. Crit Care Med 2017. 